\title{
EDITORIAL
}

\section{Health advocacy in urology training}

\section{Laurence Klotz}

Editor-in-Chief

CUAJ

The CUA/ wishes to join the international urology community in expressing our sense of loss over the premature death of Dr. Martin Resnick, the editorin-chief of the Journal of Urology, and past president of the AUA. Martin was a urologic man of all seasons, and his influence on our specialty was pervasive. He was a great friend of Canadian urology. Our condolences and best wishes to his family, friends and colleagues.
$\mathrm{T}$ he article by Michael Leveridge and colleagues ${ }^{1}$ raises an issue that is not often addressed in the urology literature, and should provoke some discomfort and discussion. Every physician involved in resident training and evaluation is aware that, about 10 years ago, the Royal College redefined the goals of training beyond the acquisition of expertise in a specialty. Six additional roles were added to that of medical expert, including health advocate, communicator, collaborator, manager, scholar and professional. Resident evaluations now require the resident's performance in each of these areas to be determined on a regular basis. For residents working hard to master surgical and clinical skills and basic and clinical sciences, the challenge represented by the acquisition of expertise in these other 6 roles, and (perhaps even more critically) the need to demonstrate that the skill sets involved have been acquired, is large.

One of these roles, the scholar (i.e., researcher), has been accepted for 100 years as an important one in surgery. Canadian residents are encouraged to obtain some research experience, and indeed this a requirement of some programs. However, it is likely that most urologists involved in resident training believe that the teaching involved in the 5 roles beyond medical expert and scholar occurs implicitly, by example, rather than by explicit instruction. We teach communication skills by being good communicators; we demonstrate collaborator skills by collaborating; and so on.

The article by Leveridge and colleagues confirms that explicit training in health advocacy is rare in residency training and that active participation in health advocacy projects is virtually nonexistent. The Royal College presumably considers that this is a deficiency. But is it? Becoming a medical expert involves the acquisition of multiple skill sets. Five years of residency training after medical school is a short time to develop proficiency. Would formal training in the other 5 roles, beyond expert and scholar, represent a dilution of the focus on knowledge acquisition and clinical and surgical skills? Or would resident instruction and opportunity for involvement in, for example, health advocacy, raise the level of expertise achieved in other areas? Does this matter? We welcome our readers' views on this question.

The Laval group continues their remarkable productivity in the area of cancer biomarkers. Stéphan Bolduc and colleagues ${ }^{2}$ report that urinary prostate specific antigen (PSA), and particularly the urinary to serum PSA ratio, discriminate between benign prostatic hyperplasia and prostate cancer in men with mild PSA elevation. Urinary PSA is appealing because of its availability and low cost. It would have been interesting to know how urinary PSA performs compared with free versus total ratio, or to a multi-parameter nomogram approach incorporating other risk factors. We look forward to more evidence regarding the utility of this assay.

\section{References}

1. Leveridge M, Beiko D, Wilson JWL, et al. Health advocacy training in urology: a Canadian survey on attitudes and experience in residency. CUAJ 2007;1:363-9.

2. Bolduc S, Lacombe L, Naud A, et al. Urinary PSA: a potential useful marler when serum PSA is between $2.5 \mathrm{ng} / \mathrm{mL}$ and $10 \mathrm{ng} / \mathrm{mL}$. CUAJ 2007;1:377-81. 